\title{
Un pas vers le traitement par transfert de gène de déficits immunitaires combinés sévères ?
}

Le transfert de gène dans les cellules souches hématopoḯtiques reste chez l'homme une approche décevante [1]. En effet, les vecteurs actuellement disponibles ne permettent d'obtenir l'expression du transgène que dans une très faible fraction des progéniteurs hématopoïétiques $\left(\mathrm{m} / \mathrm{s} \mathrm{n}^{\circ} 3\right.$, vol. 14, p. 356). Cette restriction limite actuellement la possible utilisation clinique du transfert de gènes aux quelques maladies héréditaires dans lesquelles le transfert du gène thérapeutique est susceptible de procurer un considérable avantage sélectif aux cellules transduites. C'est le cas des déficits immunitaires héréditaires combinés sévères dans lesquels le déficit génétique provoque un défaut de prolifération de précurseurs lymphocytaires. Les résultats obtenus dans les essais cliniques de transfert de gènes concernant le déficit en adénosine désaminase n'ont pas été sur ce plan totalement concluants, sans doute en raison du maintien éthiquement justifié d'un second traitement sous forme d'injection hebdomadaire intramusculaire de l'enzyme déficiente [2].

K.D. Bunting et al. démontrent dans un modèle murin, pour la première fois, la correction d'une autre forme de déficit immunitaire combiné sévère par thérapie génique [3]. Il s'agit du déficit en protéine-tyrosine kinase JAK-3 responsable d'un défaut profond de différenciation des lymphocytes T, NK et, dans une moindre mesure, B. Le déficit en protéine tyrosine kinase JAK-3 est responsable d'environ $1 / 4$ des formes humaines de déficit immunitaire combiné sévère $\left(\mathrm{m} / \mathrm{s} n^{\circ} 2\right.$, vol. 12, p. 265). L'équivalent murin a été produit par inactivation du gène par la technique de recombinaison homologue. Le travail récemment publié montre que l'infection de progéniteurs hématopoïétiques à l'aide d'un vecteur rétroviral contenant le gène de JAK-3 permet de restaurer en quelques semaines chez les souris déficientes une lymphopoïèse $\mathrm{T}$ et $\mathrm{B}$ normale. Les lymphocytes présents sont fonctionnels; en particulier, ils sont capables de développer une réponse anticorps après immunisation. La persistance de lymphocytes en nombre approximativement voisin de la moitié de la normale a été observée dans le sang pendant une période d'observation de 7 mois. Aucun effet secondaire n'a été observé chez les souris ainsi traitées. Fait remarquable, un avantage sélectif des lymphocytes transduits a pû être démontré dans la mesure où la fraction de lymphocytes transduits dans le sang est très supérieure à la fraction de cellules myéloïdes transduites. Ces résultats sont importants puisqu'ils démontrent la faisabilité d'une correction in vivo d'un déficit immunitaire combiné sévère par transfert de gène dans les cellules souches hématopoiétiques et, pour la première fois, démontre in vivo l'existence effective d'un avantage sélectif conféré aux cellules transduites.

Il s'agit donc d'une étape significative vers l'utilisation clinique de cette méthode. Cependant, la transposition automatique à l'homme ne peut en être immédiatement déduite. En effet, dans cette étude, les souris receveuses de cellules souches hématopoiétiques génétiquement modifiées, ont été irradiées de façon létale. Il est difficile d'envisager une telle thérapeutique chez des enfants atteints de déficit immunitaire combiné sévère compte tenu des risques inhérents à une telle irradiation dans ce contexte. On ne peut non plus extrapoler directement de la souris à l'homme l'efficacité d'un transfert de gène dans les cellules souches hématopoiétiques en raison de la plus faible efficacité de transfert observée chez l'homme. Enfin, les risques éventuels liés à une possible surexpression de la tyrosine-kinase JAK-3 impliquée dans les processus d'induction de prolifération cellulaire pourraient être éventuellement délétères. Cependant, à juste titre, les auteurs de cet article indiquent que cette protéine n'est activée que par la fixation de ligand au récepteur de cytokines c auquel JAK-3 est associée. Malgré ces limites, les résultats présentés dans cet article permettent de franchir une étape importante en vue d'une application clinique du traitement par transfert de gènes dans les déficits immunitaires combinés sévères, non seulement du déficit en JAK-3 mais aussi d'un déficit immunitaire voisin, le déficit immunitaire combiné sévère lié à l'X, résultant de mutations de la sous-unité $\gamma_{c}$ commune à de nombreux récepteurs de cytokines auxquelles la protéine JAK3 s'associe physiologiquement [4].

A.F.

1. Bagnis C, Chabannon C, Mannoni P. Transfert de gènes dans les cellules hématopoïétiques : obscur objet du désir de voir et de manipuler la vraie cellule souche? Med Sci 1996; 12: 60-3.

2. Valère T. Thérapie génique: le point sur les essais cliniques. Med Sci 1996; 12: 73-83.

3. Bunting KD. Restoration of lymphocyte function in JANUS kinase 3- deficient mice by retroviralmediated gene transfert. Nat Med 1998; 4: 58-64.

4. Hivroz C, Le Deist F, Fischer A. Le déficit en tyrosine kinase ZAP-70: un modèle de déficit immunitaire héréditaire pour l'analyse de l'activation et de la différenciation des lymphocytes $\mathrm{T}$. Med Sci 1995 ; 11 : 268-72. 